\title{
Streammare l'opera lirica. Gli esperimenti di digital liveness del teatro musicale contemporaneo
}

\author{
Laura Pernice \\ Università degli Studi di Catania
}

\begin{abstract}
La stagione pandemica del «lockdown theatre» (Gemini et al. 2021) ha avuto un impatto dirompente sulle procedure materiali ed evenemenziali di produzione degli spettacoli, e sulle loro modalità di fruizione da parte dei pubblici, "traslocate" dallo spazio fisico dei luoghi teatrali a quello smaterializzato dei media elettronici. In questo contesto inedito l'opera lirica ha potenziato la sua naturale vocazione alla multimodalità, nel segno di una «fenomenologia dello streaming» (Galla 2020) che ha trasformato la condizione di liveness del teatro musicale e performativo. Attraverso una mirata ricognizione dei più recenti e significativi esperimenti di mediatizzazione della performance operistica, tra cui i cartelloni di opere in streaming introdotti dalle istituzioni musicali (il Teatro digitale dell'Opera di Roma; \#operaonthesofa del Regio di Torino, etc.) e le creazioni originali concepite per la fruizione online/televisiva (II barbiere di Siviglia e La traviata di Mario Martone; A riveder le stelle di Davide Livermore; Il crepuscolo dei sogni di Johannes Erath; Alienati del Teatro Coccia), il contributo riflette sui gradienti di digital liveness dell'opera in video, sia in diretta che in differita; mettendo in relazione i presupposti epistemologici sulla liveness con la distintiva postura spettatoriale richiesta dallo spettacolo lirico, divisa tra «mimesi e astrazione» (Gallarati 2007) e tra i diversi livelli di ricezione attivi, che l'attuale fenomenologia dello streaming sta nettamente ridefinendo.
\end{abstract}

The pandemic season of «lockdown theatre» (Gemini et al. 2021) had a disruptive impact on the material and evenemential procedures of production of performances, and on the way they are received by audiences, "moved" from the physical space of theatres to the dematerialized space of electronic media. In this inedited context, opera has strengthened its natural vocation for multimodality, in the sign of a «phenomenology of streaming» (Galla 2020) that has transformed the condition of liveness of musical and performing theatre. Through a targeted reconnaissance of the most recent and significant experiments in the mediatisation of opera performance, including the streaming opera programs introduced by musical institutions (the Teatro Digitale of the Opera di Roma; \#operaonthesofa of the Regio di Torino, etc.), and the original creations conceived for online/television fruition (II barbiere di Siviglia and La traviata by Mario Martone; A riveder le stelle by Davide Livermore; /l crepuscolo dei sogni by Johannes Erath; Alienati by Teatro Coccia), the paper reflects on the gradients of digital liveness of opera on video, both live and deferred; by relating the epistemological premises on liveness with the distinctive spectatorial posture required by opera performances, divided between "mimesis and abstraction" (Gallarati 2007) and between the different levels of audience reception, which the current phenomenology of streaming is clearly redefining.

\section{Parole chiave/Key Words}

Opera lirica; digital liveness; meditizzazione teatrale; streaming; ricezione operistica.

Opera; digital liveness; theatre mediatisation; streaming; opera reception. 


\section{La liveness teatrale tra ontologia e mediatizzazione}

Sarà ricordato come il sold out più veloce de La Scala la vendita lampo dei biglietti per il Macbeth di Verdi diretto da Riccardo Chailly con la regia di Davide Livermore, che il 7 dicembre 2021 inaugurerà la stagione lirica scaligera. Sono bastate pochissime ore, infatti, per esaurire la disponibilità di posti nella sala del Piermarini per assistere al debutto dello spettacolo. La straordinarietà di tale accaparramento denota la voglia del pubblico di tornare a La Scala per il tradizionale appuntamento di Sant'Ambrogio dopo la chiusura del teatro nello scorso anno, ma, osservata in un'ottica più specifica, indica anche un desiderio di socialità e condivisione fisiche, di riaggregazione di quella "comunità estetica" ${ }^{1}$, temporanea, transeunte, ma basata sul contatto diretto, che si costituisce sull' «asse platea-scena»².

La centralità dell'interazione faccia a faccia sull'asse platea-scena (a cui si aggiunge la relazionalità diffusa sull'asse platea-platea) come specificità socio-antropologica della comunicazione teatrale è stata ampiamente trattata dagli studi teatrologici del Novecento, al punto che la stessa determinazione scientifica della disciplina poggia su questo preciso "cognitive turn": il riconoscimento dello statuto di differenza del teatro rispetto agli altri sistemi sociali e comunicativi, oggettivato dalla sua congenita composizione di linguaggi diversi $^{3}$ ma soprattutto dalla co-presenza spazio-temporale di attori e spettatori, dalla contemporaneità di produzione e consumo, e dunque dall'immediata "risposta estetica» ${ }^{4}$ del pubblico legata ai modi di fruizione e agli effetti emotivo-intellettuali provocati dall'evento. L'idea che il teatro è "un avvenimento che esiste solo entro uno spazio comune e un tempo condiviso tra attori e spettatori» è stata un'agnizione epistemologica fondamentale per l'evolversi della teatrologia novecentesca, per il suo upgrade postmoderno da «storia della letteratura drammatica e storia degli eventi spettacolari ${ }^{5}$, e pertanto delle loro specifiche

\footnotetext{
${ }^{1}$ Cfr. G. Vattimo, La società trasparente, Garzanti, Milano 2000.

${ }^{2}$ Cfr. P.C. Rivoltella, La differenza comunicativa del teatro. Aspetti teorici e implicazioni educative, in "Comunicazioni Sociali», n. XVIII, 1996, pp. 5-29.

${ }^{3} \mathrm{O}$ meglio, con Marco De Marinis, di «drammaturgie parziali: quella dell'autore, quella degli attori, del regista, dello scenografo, del musicista». Cfr. M. De Marinis, In cerca dell'attore. Un bilancio del Novecento teatrale, Bulzoni, Roma 2000, p. 29.

${ }^{4}$ Cfr. W. Iser, L'atto della lettura. Una teoria della risposta estetica [1978], il Mulino, Bologna 1987.

${ }^{5}$ L. Locatelli, Lo spettatore, in A. Cascetta, L. Peja (a cura di), Ingresso a teatro. Guida all'analisi della drammaturgia, Le Lettere, Firenze 2003, p. 99.
} 
modalità di enunciazione/ricezione - comportamentali, cognitive, emotive, etc. - basate sull'evenemenzialità dell'hic et nunc.

L'eccezionale rapidità del sold out de La Scala per la «prima che torna in presenza», decantata da tutti i giornali tra l'entusiasmo per «la riapertura dei teatri a capienza piena» e il "memento" dell' «obbligo di Green Pass o di tampone negativo al Covid per accedere al teatro ${ }^{6}$, ci sembra uno spunto utile da cui partire per riflettere sulla specificità della comunicazione teatrale (intesa appunto come co-presenza fisica di attori e spettatori e identità delle categorie spazio-temporali) e sulle sue radicali trasformazioni fenomenologiche, che negli ultimi anni sono state prodotte dallo sviluppo delle tecnologie mediali, e negli ultimi mesi sono state (iper) accelerate dal loro utilizzo massivo a seguito dell'avvento della pandemia.

Riassumendo un'ampia prospettiva analitica che, soprattutto dagli anni Novanta del secolo scorso guarda agli effetti del cambiamento socio-comunicativo su quello performativo, e viceversa, sappiamo che il rapporto fra teatro e media storicamente si è divaricato su due grandi versanti: «Se da un lato, tale rapporto ha caratterizzato la ricerca della specificità teatrale nell'opposizione con i media, dall'altro lato la scena novecentesca si è caratterizzata come contesto di sviluppo delle arti multimediali, operando in direzione di una progressiva assimilazione di formati e linguaggi mediali» ${ }^{7}$. In questo scenario fortemente ambivalente, tuttavia, la pervicace e pervasiva mediatizzazione del sociale ${ }^{8}$, ossia la costruzione dei sistemi socio-comunicativi attraverso pratiche e protocolli propri dei media, ha spinto con forza sulla

\footnotetext{
${ }^{6}$ A epitome di un'ampia rassegna stampa si veda D. Solito, Scala, sold out in poche ore i biglietti per la Prima del 7 dicembre, in «la Repubblica», 27 ottobre 2021, https://milano.repubblica.it/cronaca/2021/10/27/news/prima_della_scala_tutto_esaurito_macbeth_di_liverm ore-324005002/ (ultimo accesso 25/11/2021).

${ }^{7}$ L. Gemini, S. Brilli, F. Giuliani, Il dispositivo teatrale alla prova del Covid-19. Mediatizzazione, liveness e pubblici, in "Mediascapes journal», n. 15, 2020, p. 46. Sull'intensa relazione tra teatro e media si veda L. Gemini, L'incertezza creativa. I percorsi sociali e comunicativi delle performance artistiche, FrancoAngeli, Milano 2003; A. Balzola, A.M. Monteverdi, Le arti multimediali digitali, Garzanti, Milano 2004; A.M. Monteverdi, Nuovi media, nuovo teatro. Teorie e pratiche tra teatro e digitalità, FrancoAngeli, Milano 2011; A. Pizzo, Neodrammatico digitale: Scena multimediale e racconto interattivo, Accademia University Press, Torino 2013; A. Amendola, V. Del Gaudio (a cura di), Teatro e immaginari digitali. Saggi di sociologia dello spettacolo multimediale, I gechi, Salerno 2017; A.M. Monteverdi, Leggere uno spettacolo multimediale. La nuova scena tra video mapping, interaction design e Intelligenza Artificiale, Dino Audino, Roma 2020.

${ }^{8}$ Declinata come meccanismo pervasivo della società contemporanea, la mediatizzazione è considerata un vero e proprio meta-processo insieme a globalizzazione, individualizzazione e commercializzazione; cfr. F. Krotz, The Meta-Process of "Mediatisation" as a Conceptual Frame, in "Global media and communication», vol. 3, n. 3, 2007, pp. 256-260. In termini analitici essa si articola su due livelli: da un lato un processo di cambiamento sociale attualizzato dai media, dall'altro una serie di funzioni svolte dai media nella società; cfr. W. Schultz, Reconstructing Mediatisation as an Analytical Concept, in «European Journal of Communication», vol. 19 n. 1 , 2004, pp. 87-101.
} 
forma teatrale, determinando una cospicua serie di "effetti" sulla sua fenomenologia - transmedialità, rimediazione, hypermediacy, serializzazione, etc. -, che vertono tutti sulla netta ridefinizione della condizione di liveness.

Da sempre consustanziale alle arti performative - a partire dal modello dell'interazione faccia a faccia -, la liveness, l'esperienza del "dal vivo", per molto tempo è stata considerata la caratteristica precipua del medium teatrale, la cifra della sua forma estetica e comunicativa fondata sulla simultaneità produzione/consumo e sulla copresenza fisica spettacolo/spettatore; in altri termini, secondo la classica ontologia degli studi teatrali e dei performance studies, essenzialmente «liveness è la forma della comunicazione dal vivo basata sull'hic et nunc» ${ }^{9}$.

Si deve al noto studio di Philip Auslander ${ }^{10}$, interamente dedicato alla nozione di liveness nella dimensione performativa, un decisivo ampliamento concettuale di questa peculiare esperienza; lo studioso, infatti, introduce una distinzione tra «classical liveness», determinata da "physical co-presence of performers and audience; temporal simultaneity of production and reception; experience in the moment ${ }^{11}$, e liveness "historically contingent» che, "with the advent of broadcast technologies - first radio, then television», si ridefinisce come "live broadcast". [...] The liveness of the experience of listening to or watching the recording $»^{12}$.

La forza della riarticolazione proposta da Auslander sta nel fatto che le due accezioni di liveness non sono considerate antitetiche, giacché entrambe possiedono una valenza primariamente affettiva: l'esperienza di guardare una performance registrata dal vivo o di assistere alla sua diretta televisiva provoca un senso di partecipazione emotiva e di vicinanza temporale, a tutti gli effetti una relazione vicaria con l'audience "reale" di quella data performance, tale da supplire alla mancata co-presenza fisica. In più, come spiega efficacemente Vincenzo Del Gaudio,

\footnotetext{
${ }^{9}$ L. Gemini, Liveness: le logiche mediali nella comunicazione dal vivo, in "Sociologia della Comunicazione», $\mathrm{n}$. 51, 2016, p. 45.

${ }^{10}$ P. Auslander, Liveness: Performance in a Mediatized Culture [1999], Routledge, Oxon 2008.

11 Ivi, p. 61.

12 Ivi, p. 60.
} 
Per Auslander non esiste una coppia oppositorum tra i due poli bensì una relazione reciproca tipica del nuovo universo mediale [...], relazione in base alla quale l'evento live è sempre più contaminato dall'evento mediale sin nelle sue fondamenta. Si viene così a creare uno spazio dove la tecnologia digitale modifica le funzioni della liveness restituendo [...] vari livelli di liveness e vari strati di mediatizzazione che sono sempre aperti e spesso convivono nello stesso evento scenico. ${ }^{13}$

Proponendo la liveness come condizione fenomenologica storicamente contingente, e pertanto soggetta a trasformazioni e riarticolazioni legate alla mediazione tecnologica della relazione teatrale, più che come caratteristica insita nel medium, unicamente inscritta nelle sue «basic conditions» di «performers and audience physically and temporally co-present ${ }^{14}$, la traiettoria di Auslander segna uno scarto notevole rispetto alla classica linea teatrologica, e si pone a fondamento di un ampio e crescente ambito di studi che analizza i diversi "gradienti" ${ }^{15}$ di liveness «lungo il continuum delle performance mediatizzate che procede dalle performance multimediali alle performance trasferite sui supporti mediali e online ${ }^{16}$.

L'eterogeneità di questo orizzonte analitico - che convoglia ricerche di theater studies, di sociology and media studies, di audience and reception studies - trova nell'ultimo fronte della riflessione di Auslander, ossia la declinazione di digital liveness degli spettacoli mediatizzati, un nodo tematico condiviso e cruciale, ormai del tutto ineludibile. È evidente, infatti, che lo sviluppo della mediatizzazione (live e non) di eventi teatrali, oltre a ridisegnare la fisionomia produttiva, distributiva e fruitiva delle performing arts ha ulteriormente modificato la liveness teatrale, rendendola un'esperienza cognitivopercettiva di digital liveness. Tale esperienza, situandosi nelle pratiche del live stream e della fruizione in differita su piattaforme online (né qui né ora), può trascendere il real time tanto quanto la co-presenza fisica, riarticolandosi in una «specific relation between self and other, a particular way of "being involved with something". The experience of liveness results from our conscious act of grasping virtual entities as live in response to

\footnotetext{
${ }^{13}$ V. Del Gaudio, Modelli di serialità teatrale: Pratiche per un approccio mediologico al teatro a partire dai modelli seriali della Tragedia Endogonidia della Sociétas Raffaello Sanzio e Ada. Una cronaca familiare di Fanny \& Alexander, in «Mediascapes journal», n. 8, 2017, p. 257.

14 P. Auslander, op. cit., 2008, p. 60.

15 L'acuta definizione di "gradienti di liveness" volta a indicare la semantica mobile e complessa di tale concetto si deve a G. Boccia Artieri, L. Gemini, Per un live dei corpi a distanza, in "Doppiozero», $1^{\circ}$ maggio 2020, https://www.doppiozero.com/materiali/un-live-dei-corpi-distanza (ultimo accesso 25/11/2021).

${ }^{16}$ L. Gemini, op. cit., 2016, p. 54.
} 
the claims they make on us» ${ }^{17}$. Osservato in quest'ottica, chiarisce bene Laura Gemini, «la liveness [...] non è una caratteristica dell'oggetto, né un suo effetto ma è a sua volta una forma dell'interazione prodotta attraverso l'engagement del fruitore con l'oggetto e dalla sua volontà di accettare che l'artefatto si presenti come live ${ }^{18}$.

Nel tornare ad analizzare il senso di "dal vivo" Auslander mette in campo una più articolata ipotesi di realizzazione dell'esperienza teatrale: sganciata dall'esclusiva e ontologica qualità del qui e ora, dinamicamente connessa alle trasformazioni dei vissuti mediali di chi crea e di chi fruisce le produzioni artistiche, e dunque scaturente da un nuovo patto spettatoriale che, in un reciproco "tendersi" tra spettacolo e pubblico entro lo spazio fluido dell'attuale mediasfera ${ }^{19}$, poggia sulla mutuale intenzionalità di darsi, oggetto e soggetto dell'esperienza, a un intenso "processo di co-determinazione reciproca di matrice relazionale ${ }^{20}$.

È alla luce di tali presupposti epistemologici sulla liveness che occorre guardare alla condizione mediale degli spettacoli, specialmente in merito alla recente "ondata" di performance digitali prodotta dalla chiusura forzata dei luoghi teatrali, e a quella forma d'arte ritenuta esteticamente tra le più complesse e plurimateriche ${ }^{21}$, allo stesso tempo segnatamente musicale e performativa, che è l'opera lirica. Dopo tale introduzione preparatoria, dunque, restringeremo la riflessione sullo specifico gradiente di digital liveness dell'opera trasmessa e fruita in video - sia in diretta che in differita -, mettendo in relazione le acquisizioni teoriche fin qui esposte con la distintiva postura spettatoriale richiesta dallo spettacolo lirico, divisa tra «mimesi e astrazione $»^{22}$ e tra i diversi livelli di ricezione attivi (partitu-

\footnotetext{
${ }^{17}$ P. Auslander, Digital liveness: a historico-philosophical perspective, in «PAJ: A Journal of Performance and Art», vol. 34, n. 3, 2012, p. 10.

18 L. Gemini, op. cit., 2016, p. 52.

${ }^{19}$ Coniato dallo studioso Régis Debray il termine mediasfera indica l'ambiente dei media che caratterizza un'epoca. Cfr. R. Debray, Vita e morte dell'immagine. Una storia dello sguardo in Occidente [1992], II Castoro, Milano 2010.

${ }^{20}$ L. Gemini, op. cit., 2016, p. 52.

${ }^{21}$ Cfr. N. Till (a cura di), Opera Studies, Cambridge University Press, Cambridge 2012; S. O'Neil, J. Edelman, J. Sloboda, Opera Audiences and Cultural Value: A Study of Audience Experience, in "Creativeworks London Working Paper», n. 2, 2014.

${ }^{22}$ Cfr. P. Gallarati, Mimesi e astrazione nella regia del teatro musicale, in R. Alonge (a cura di), La regia teatrale: specchio delle brame della modernità, Edizioni di Pagina, Bari 2007, pp. 175-188.
} 
ra, drammaturgia, messa in scena), che nell'opera in streaming moltiplicano sensibilmente la già spiccata «complessificazione del concetto e delle pratiche della liveness» ${ }^{23}$.

\section{L'opera lirica nella fenomenologia dello streaming}

La lunga stagione pandemica dei teatri chiusi e inaccessibili al pubblico è ormai giunta al termine, e da alcune settimane lo spettacolo dal vivo ha raggiunto il traguardo della capienza piena delle sale. Tuttavia il periodo appena attraversato, nelle sue progressive articolazioni di «lockdown theatre, theatre of distancing and theatre of empty seats» ${ }^{24}$ ha avuto un impatto dirompente sulle procedure materiali ed evenemenziali di produzione degli spettacoli, e sulle loro modalità di fruizione da parte dei pubblici, "traslocate" dallo spazio fisico dei luoghi teatrali a quello smaterializzato dei media elettronici.

Sebbene la deriva tecnomediale e il processo di virtualizzazione ${ }^{25}$ da essa sospinto agiscano già da tempo sulla forma-teatro, per cui da oltre un decennio si assiste all'incremento del webcasting ${ }^{26}$ di eventi spettacolari, ed esiste un'ampia letteratura critica che si è occupata del fenomeno - specie nel mondo anglosassone ${ }^{27}$, è palese lo spread prodotto dalla pandemia sulla produzione e il consumo di spettacolo digitale, tant'è che secondo un recente studio americano non si dovrebbe parlare di un "before and after" quanto piuttosto di un "before and faster": I'emergenza pandemica ha accelerato delle trasformazioni già in atto, e in questo senso durante il lockdown i contenuti teatrali mediatizzati hanno svolto un ruolo importante nel mantenere attivo il contatto con il pubblico e nel sostenere il suo benessere nonostante la crisi ${ }^{28}$.

\footnotetext{
${ }^{23}$ L. Gemini, op. cit., 2016, p. 48.

${ }^{24}$ G. Boccia Artieri, L. Gemini, S. Brilli, F. Giuliani, The reinvention of theatre space during Covid-19: Analysis of the Italian case. Articolo gentilmente concesso dagli autori in anteprima, attualmente in corso di pubblicazione negli atti del Simposio Internazionale Borštnikovo srečanje 2021 a cura di Tomaž Krpič.

${ }^{25}$ Su questi concetti si veda L. Gemini, op. cit., 2003, pp. 43-52.

${ }^{26}$ Con webcasting si identifica l'architettura di trasmissione di contenuti tipica del broadcasting (da uno a molti) erogata attraverso le reti digitali. Cfr. J. Whittaker, The Cyberspace Handbook, Routledge, London 2004.

${ }^{27}$ Tra i contributi più significativi si veda l'analisi promossa dall'Art Council of England nel 2016: B.K. Reidy, B. Schutt, D. Abramson, A. Durski, From Live-to-Digital: Understanding the Impact of Digital Developments in Theatre on Audiences, Production and Distribution, London, AEA Consulting for Arts Council England, UK Theatre and Society of London Theatre 2016, https://www.artscouncil.org.uk/sites/default/files/downloadfile/From_Live_to_Digital_OCT2016.pdf (ultimo accesso 25/11/2021).

${ }^{28}$ Cfr. K. Edelman, B. Hurley, N. Gangopadhyay, Digital media trends survey, 14th edition, a cura di Deloitte's Technology, Media \& Telecommunications practice, https://www2.deloitte.com/lu/en/pages/technologymedia-and-telecommunications/articles/digital-media-trends-survey.html (ultimo accesso 25/11/2021).
} 
La proliferazione di spettacoli in streaming durante l'ultimo anno e mezzo, a ben guardare, si connota in modo ambivalente: in parte ha suturato la ferita dei teatri privati degli spettatori grazie alla fibra strumentale della tecnologia, altresì ha consentito di estendere le possibilità di visione ad audience materialmente impensabili nelle sale in presenza; di contro, però, ha determinato una forte disuguaglianza tra chi ha potuto investire nella sfida tecnologica e chi per ragioni di disponibilità economica ne è rimasto escluso: in tal senso le Fondazioni lirico-sinfoniche italiane hanno beneficiato di una congiuntura ottimale, frutto dei maggiori finanziamenti pubblici erogati attraverso il FUS - il Fondo Unico per lo Spettacolo - e, nel contempo, della drastica riduzione dei costi per le produzioni digitali. Certamente l'effetto più considerevole di tale «fenomenologia dello streaming ${ }^{29}$ ha riguardato la dimensione della liveness, portando il teatro a rinegoziare ancora una volta la propria natura attorno a un regime di evenemenzialità impersonale e tecnicamente riproducibile, il solo consentito a fronte della coatta spoliazione della presenza soggettiva dei corpi.

Nel perimetrare lo scenario inedito dell'opera lirica al tempo della pandemia il primo dato interessante riguarda la risposta rapida e volitiva che gli enti lirici italiani hanno saputo dare ai decreti di chiusura volti al contenimento dell'emergenza.

La prima proposta di "streammare" i melodrammi è arrivata dal Teatro Massimo di Palermo che, al grido perentorio di "Non vi lasceremo senza musica!» ${ }^{30}$, già il 9 marzo 2020 ha varato sulla propria web tv un'ampia programmazione di spettacoli ripresi dai suoi maggiori successi degli ultimi anni. Dallo "start" dell'ente lirico siciliano una dopo l'altra le principali istituzioni musicali della penisola hanno presentato i loro cartelloni alternativi, in un crescendo di proposte tanto diversificate quanto subordinate alla digitalizzazione dei patrimoni documentari. I teatri che avevano investito nella politica di creazione di archivi digitali hanno acquisito di default un vantaggio competitivo rispetto a chi non aveva perseguito lo stesso obiettivo, potendo offrire alle "platee da casa" ampi ventagli di titoli comprensivi del grande repertorio così come di rarità d'epoca ormai dimenticate, adesso riportate allo sguardo dalla proiezione atemporale dello streaming.

\footnotetext{
${ }^{29}$ Cfr. C. Galla, Opera lirica: fenomenologia dello streaming, in «Doppiozero», 12 dicembre 2020, https://www.doppiozero.com/materiali/opera-lirica-fenomenologia-dello-streaming (ultimo accesso 25/11/2021).

${ }^{30} \mathrm{Cfr}$. https://www.teatromassimo.it/archivio/notizie/non-vi-lasciamo-senza-musica.html (ultimo accesso 25/11/2021).
} 
Tra le iniziative più rilevanti attivate durante il lockdown ricordiamo il Teatro digitale dell'Opera di Roma, un nutrito cartellone di concerti, opere e balletti diffusi sia in streaming che in live-streaming sul canale YouTube dell'ente romano; il palinsesto nonstop del San Carlo di Napoli lanciato con l'hashtag \#stageathome, che sulla neonata web tv del teatro ha articolato un'ardita offerta di opera in streaming 24 ore su 24; la ricca rassegna \#operaonthesofa del Regio di Torino che, distillata in precisi appuntamenti quotidiani "all'ora dell'aperitivo» - tutti i giorni alle 18 - ha spaziato dalle produzioni della stagione in corso a quelle passate fino alle riprese inedite di prove generali, presentate con l'ammiccante slogan «Noi portiamo la musica e voi, se volete, aprite una bottiglia di vino ${ }^{31}$. A tali cartelloni digitali, poi, va aggiunto il cospicuo bagaglio video-musicale di trenta produzioni registrate tra il 2008 e il 2019 - in gran parte inedite per il web - che La Scala ha ceduto alla Rai per renderle gratuitamente fruibili sulla piattaforma streaming RaiPlay, instaurando così una sinergia importante tra la principale Fondazione liricosinfonica italiana e l'ente del Servizio Pubblico radiotelevisivo e multimediale; e l'iniziativa promossa dai maggiori enti lirici dell'Emilia-Romagna con il sostegno dell'Assessorato alla Cultura, che, proprio a ridosso dei decreti di chiusura hanno inaugurato OperaStreaming, il primo portale regionale interamente dedicato allo streaming operistico che, come si legge sul suo sito, estende i propri propositi di divulgazione ben oltre la contingenza del lockdown, puntando a fornire sul lungo periodo «un servizio rivolto a fasce di pubblico svantaggiate nella fruizione delle attività delle sale teatrali» ${ }^{32}$.

Sebbene siano stati utili all'audience engagement soprattutto rivolto alle giovani generazioni (confermando i dati sul rinnovo dei pubblici della lirica attraverso la fruizione digitale già acquisiti prima del Covid $)^{33}$ tali cartelloni in streaming, tuttavia, nella maggior parte dei casi hanno proposto registrazioni audiovisive di spettacoli storici o delle stagioni passate, ossia oggetti mediali che costituiscono un documento indiretto di ciò che è accaduto sui palcoscenici, "soltanto" una testimonianza artistica o traccia mnestica in differita, e pertanto "a bassa intensità di liveness". Con tale considerazione ci riferiamo al fatto che,

\footnotetext{
${ }^{31}$ Cfr. https://www.teatroregio.torino.it/news/operaonthesofa (ultimo accesso 25/11/2021).

${ }^{32}$ Cfr. https://operastreaming.com/il-progetto/ (ultimo accesso 25/11/2021).

${ }^{33} \mathrm{Ci}$ riferiamo in particolare alle indagini pubblicate dal report realizzato con la consulenza scientifica di Laura Gemini, Stefano Brilli e Francesca Giuliani Il pubblico dello spettacolo nelle Marche. Report di ricerca sui pubblici dello spettacolo dal vivo nelle Marche, Consorzio Marche Spettacolo, 2021, pp. 10-11.
} 
evidentemente, la fruizione di riprese registrate e riferite a un passato più o meno lontano riporta alla prima declinazione di liveness teorizzata da Auslander: a livello soggettivo e percettivo la videoregistrazione procura un senso affettivo di "dal vivo" legato al fatto che le tecnologie di visione - in questi casi le web tv dei teatri - sono esperite come live, ma ciò coinvolge primariamente l'agency individuale e collettiva del pubblico, la sua volontà di soddisfare tale esigenza affettiva, e non le logiche espressive riflessive ${ }^{34}$ delle performance trasmesse, nate per la visione in presenza, per un engagement non mediato.

Di tutt'altro spessore, invece, sono state quelle operazioni di opera in video concepite durante l'emergenza pandemica appositamente per la fruizione online e televisiva, che, consapevoli di dover affrontare una crisi di relazionalità spaziale con i pubblici forzatamente a distanza, hanno rimodulato le categorie della simultaneità trasmissione/ricezione, dell'engagement e della località con l'esperienza ${ }^{35}$ lavorando alla ricostruzione di un senso del luogo, di un tempo condiviso o di una disposizione affettiva da parte delle audience all'interno di strutture drammaturgiche $a b$ origine mediatizzate.

È proprio in questi esperimenti, a nostro avviso, che si coglie un rimodellamento della forma espressiva teatrale e dell'interazione col pubblico tale da superare il procurato senso di liveness delle videoregistrazioni classiche, approdando a un più intenso "gradiente" dell'esperienza musicale e performativa, ossia a una digital liveness frutto di autentica «co-determinazione fra affordance tecnologiche e agency di performer e pubblici» ${ }^{36}$.

\section{Le operazioni-opere di digital liveness}

Se già lo sguardo militante di Antonio Gramsci aveva individuato il carattere nazional-popolare del melodramma, che, forte di un linguaggio plurimo accessibile a tutte le classi sociali, con il proliferare dei teatri a inizio Novecento raggiungeva un pubblico sempre più vasto ${ }^{37}$, è chiaro che l'incontro dello spettacolo lirico con le tecnologie audio-

\footnotetext{
${ }^{34} \mathrm{Sul}$ «processo di reciproca determinazione, circolare e soprattutto riflessivo» tra le manifestazioni performative, il sistema sociale e la configurazione culturale su cui esse sorgono si veda L. Gemini, op. cit., 2003, pp. 22-24.

${ }^{35}$ Su questi concetti si veda M. Barker, Live at a cinema near you': How audiences respond to digital streaming of the arts, in J. Radbourne, H. Glow, K. Johanson (a cura di), The audience experience: A critical analysis of audiences in the performing arts, Intellect, Bristol 2013, pp. 15-34.

${ }^{36}$ L. Gemini, op. cit., 2016, p. 49. Ancora con Gemini specifichiamo che le affordance indicano «le opportunità di interazione che gli artefatti tecnologici possiedono in relazione alle capacità dell'artista» (L. Gemini, op. cit., 2003, p. 122). ${ }^{37}$ Cfr. A. Gramsci, Letteratura e vita nazionale [1950], Editori Riuniti, Roma 1991.
} 
visive - prima analogiche poi digitali - ha prodotto un'accentuazione notevolissima della sua costitutiva «multimodalità ${ }^{38}$, nonché della sua indole nazional-popolare, grazie alla capacità democratizzante dei media di connettere la sfera pubblica ${ }^{39}$.

Ambedue questi aspetti sono emersi con forza nel contesto inedito del «mondo flagellato dal Covid», ovvero quando "le restrizioni scattate alla fine di ottobre hanno svuotato i teatri non solo in Italia, [...] generando un benefico effetto di stimolo alla creatività in rapporto [...] alle modalità di comunicazione di un linguaggio scenico inevitabilmente molto diverso da quello abituale ${ }^{40}$. Accanto alla diffusione - che abbiamo già osservato - dei cartelIoni di opere in streaming introdotti dalle istituzioni musicali, si è verificata infatti una sorprendente accelerazione dei processi inventivi da parte degli artisti, per cui «le potenzialità live dell'online [...] sembrano trovare oggi delle importanti possibilità di attualizzazione, soprattutto della chance di normalizzazione che lo stato di necessità permette di sondare ${ }^{41}$.

Nel versante di una "pars construens» dell'opera in streaming, che può essere produttivamente intesa come "una riattivazione "per necessità" del dispositivo "spettacolo dal vivo" che tiene conto di vincoli e limiti nuovi e che spetta agli artisti declinare in possibilità creative ${ }^{42}$, si sono distinti soprattutto alcuni esperimenti, portatori di peculiarità strutturali ed estetiche che meritano attenzione.

Si è concentrata sulla reinvenzione dello spazio performativo la doppia operazione video-teatrale di Mario Martone che, in pieno lockdown, ha ideato e realizzato due allestimenti per il Teatro dell'Opera di Roma: /l barbiere di Siviglia di Gioachino Rossini visto "in debutto" su Rai3 il 5 dicembre 2020 da oltre 680.000 telespettatori, e poi inserito stabilmente nel catalogo streaming di RaiPlay, e La traviata di Giuseppe Verdi, andata in onda sulla stessa rete il 9 aprile 2021 con quasi 1 milione di telespettatori, e anch'essa poi aggiunta alla piattaforma su internet della Rai $^{43}$.

\footnotetext{
${ }^{38}$ Con multimodalità ci riferiamo all' «intreccio dei modi (da intendersi come risorse semiotiche) in diversi tipi di testo. [...] intendendo, dunque, l'opera lirica come semiosi, forma di comunicazione complessa basata sull'azione simultanea di risorse diverse e parimenti importanti». Cfr. F. Rossi, L'opera italiana: lingua e linguaggio, Carocci, Roma 2018, pp. 10-11.

${ }^{39}$ Su questo cfr. C. Balme, The theatrical public sphere, Cambridge University Press, Cambridge 2014.

${ }^{40}$ C. Galla, op. cit.

${ }^{41} \mathrm{G}$. Boccia Artieri, L. Gemini, op. cit.

42 Ibidem.

${ }^{43}$ Entrambi gli spettacoli, infatti, sono coproduzioni dell'ente lirico romano e di Rai Cultura.
} 


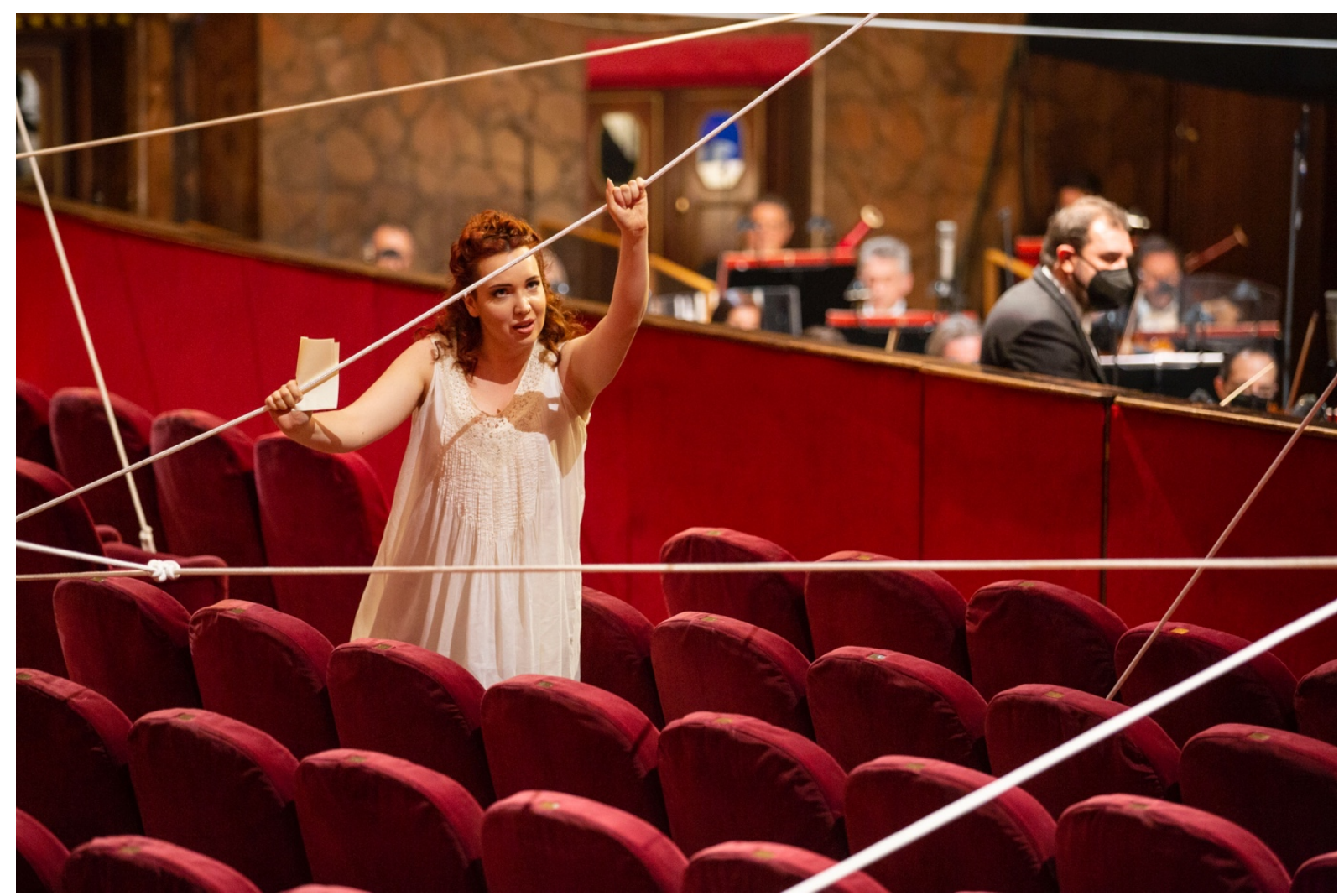

Fig. 01. Mario Martone, Il barbiere di Siviglia (2020).

Foto: Yasuko Kageyama, per gentile concessione della Fondazione Teatro dell'Opera di Roma.

Appositamente concepiti per essere trasmessi sui canali televisivi e online, entrambi gli spettacoli risultano esemplari, addirittura paradigmatici di un netto ripensamento del genere del film d'opera, che il regista napoletano ha rimodulato stilisticamente come opera-film/TV-opera ${ }^{44}$, lavorando sulla cineficazione spaziale ${ }^{45}$ della scena operistica e delle sue dinamiche attanziali.

\footnotetext{
${ }^{44}$ Superando la classificazione data dalla letteratura sui video d'opera, che individua solo tre categorie - riprese dal vivo di una rappresentazione scenica, produzioni in studio, film d'opera. Cfr. B. Large, voci Filming e Videotaping, in S. Sadie (ed.), The New Grove Dictionary of Opera, Macmillan, London 1992; e M. Veilleux, L'opera dal teatro allo schermo televisivo, in J.-J. Nattiez (a cura di), Storia della musica europea, Einaudi, Torino 2004, pp. 849-870) -, il regista francese Jean-François Jung ha produttivamente aggiunto la quarta categoria delle TVopere, ossia opere composte specificatamente per la trasmissione televisiva, aventi proprie peculiarità poetiche, estetiche ed ermeneutiche. Cfr. J.-F. Jung, Le relâchement narratif dans le système de la captation, in Opera e televisione: un problema di linguaggi, Atti del Seminario internazionale (Roma, 27-30 ottobre 1993), I.R.Te.M., Roma 1997, pp. 39-55.

${ }^{45}$ Usiamo il termine cineficazione secondo l'accezione data da Mario Verdone, il quale ne parlò come dell'assimilazione linguistica da parte del teatro di principi propriamente cinematografici. Cfr. M. Verdone, Teatro e cinema: interazioni, in C. Vicentini (a cura di), II teatro nella società dello spettacolo, il Mulino, Bologna 1983, pp. 51-62. Sulla cineficazione del medium teatrale si veda anche A.M. Monteverdi, op. cit., 2011, pp. 91-113.
} 


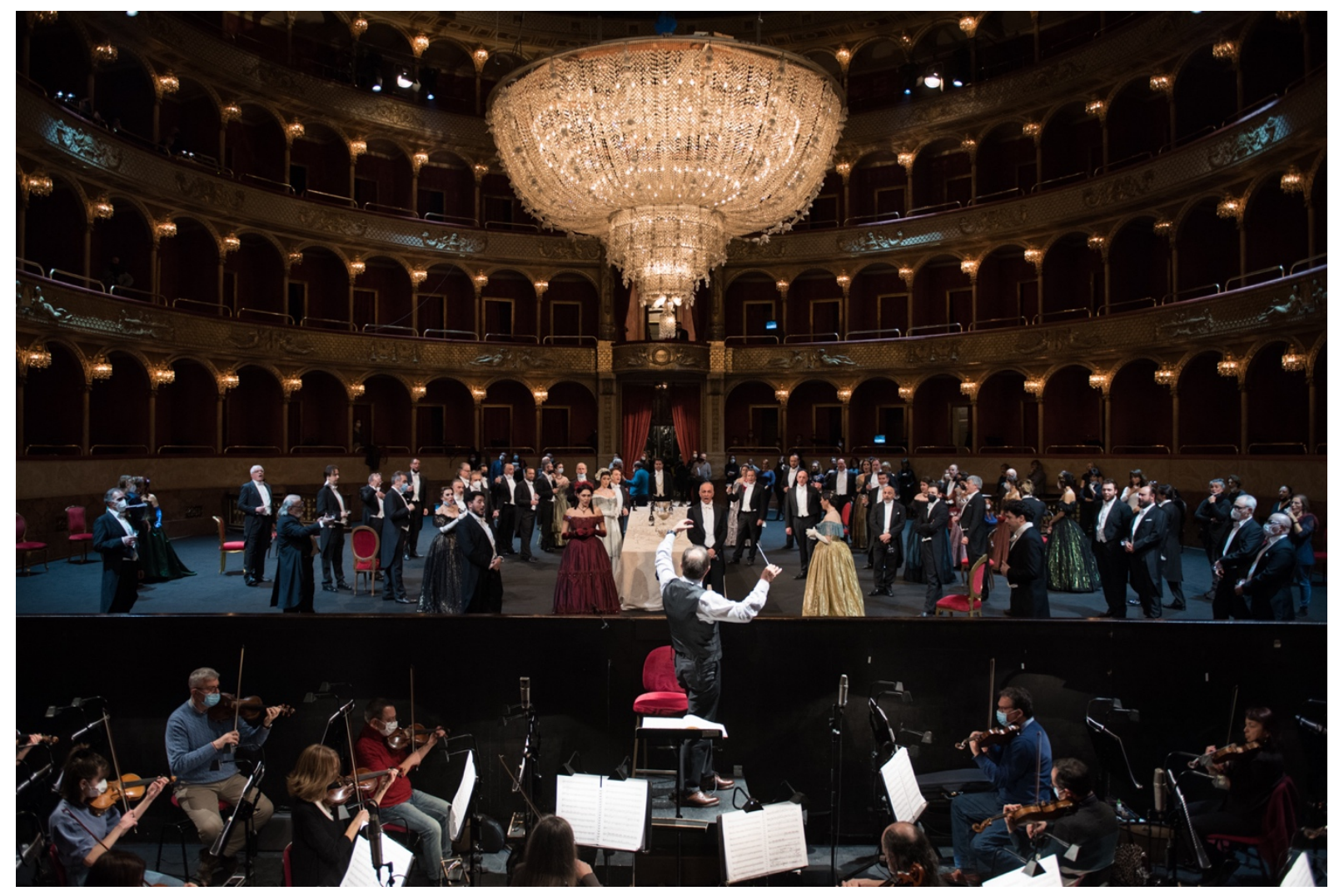

Fig. 02. Mario Martone, La traviata (2021).

Foto: Fabrizio Sansoni, per gentile concessione della Fondazione Teatro dell'Opera di Roma.

La regia intermediale ${ }^{46}$ di Martone, il quale ha contestualmente messo in scena e filmato le performance secondo un deciso modus operandi di «teatro-cinema» ${ }^{47}$, funziona in una doppia, spiazzante direzione: da una parte la designazione filmica dello spazio vuoto de /l barbiere, rappresentato e filmato nella sala deserta del Teatro Costanzi per evidenziare l'assenza del pubblico e la finzione scenica, e, dall'altra, l'occultamento cinematografico del vuoto spettatoriale e dell'artificio performativo de La traviata, ottenuto trasformando l'intera platea dell'Opera di Roma in un set realistico e site-specific.

Al di là della dirompente forza d'urto con cui questi allestimenti abbattono le convenzioni formali della messa in scena operistica, reiventandola secondo principi e procedimenti di ordine cinematografico, quel che ci interessa rilevare in questa sede è

\footnotetext{
46 Per approfondire la nozione di intermedialità intesa come interazione intersemiotica, come ricreazione/rimediazione delle convenzioni estetiche di un medium all'interno di un medium differente, si veda C. Balme, Intermediality. Rethinking the relationship between theatre and media, in «The Wis. Zeitschrift der Gesellschaft für Theaterwissenschaft», 1, 2004, pp. 1-18; e J. D. Bolter, R. Grusin, Remediation. Understanding New Media, The MIT Press, Cambridge 1999.

${ }^{47}$ Cfr. A.M. Monteverdi, op. cit., 2020, pp. 41-48.
} 
l'esaltazione della liveness teatrale che, proprio in virtù della spiccata cineficazione delle opere, si viene a produrre nell'audience domestica.

Seppure non si tratti di performance live videotrasmesse, né di spettacoli realizzati secondo la tecnica del real time film ${ }^{48}$, bensì di studiati lavori di scrittura scenica ${ }^{49}$ e di filmmaking diffusi solo post-produzione, la loro visione mediatizzata - sia televisiva che online - paradossalmente esalta la liveness operistica attraverso due "infrazioni" della canonica fruizione in sala. La prima: la platea vuota e trasformata in spazio d'azione annichilisce la dimensione spettatoriale, e laddove manca l'audience "reale" si riduce il gap e si amplifica il coinvolgimento di quella "virtuale" - giacché la stessa presenza degli astanti evidentemente denuncia uno scarto tra le due. La seconda: mentre la consueta spettatorialità della lirica implica sempre «bruschi passaggi dalla mimesi all'astrazione», ossia lo sforzo di alternare continuamente la percezione della «mimesi del gesto realistico» con quella del «senso di astrazione che si sprigiona dalla musica $»^{50}$, l'innovativa regia di Martone, nella maniera in cui inserisce il regime della rappresentazione scenica in quello della narrazione filmica, annulla l'astrazione propria della fruizione melodrammatica e la trasforma in una ricezione mimetica. Detto in altri termini, sostituisce il "patto di incredibilità" su cui poggia l'azione cantata con un "patto di plausibilità" reso possibile dall'illusione del cinema, dalla sua peculiare costruzione visiva per mezzo del montaggio.

Tanto ne /l barbiere quanto ne La traviata, infatti, «il piano cinematografico inghiotte l'azione drammatica ${ }^{51}$, il che significa che l'ópsis impersonale del cinema satura quella personale e soggettiva del teatro, corroborando la specifica mobilità mediale ${ }^{52}$ del medium teatrale, ma soprattutto rifondando il patto spettatoriale su cui si basa la liveness operistica nel segno di un'esperienza percettiva coesa, non più “biforcata” tra mimesi e astrazione. Pur ri-

\footnotetext{
${ }^{48}$ Con real time film (o live film) si intende una performance dal vivo restituita in immagine, la quale non è lavorata altrove e poi fissata su un supporto (pellicola o digitale), ma composta e mixata in tempo reale. Sull'utilizzo di questa tecnica si veda V. Valentini, Forme della presenza: performing arts e nuove tecnologie, in E. Pitozzi (a cura di), «Culture Teatrali. On Presence», n. 21, 2011, pp. 71-89; e A.M. Monteverdi, op. cit., 2020, pp. 46-47.

${ }^{49}$ Con scrittura scenica si intende un sistema di scrittura che mette in relazione tutti gli elementi linguistici del teatro. Cfr. L. Mango, La scrittura scenica. Un codice e le sue pratiche nel teatro del Novecento, Bulzoni, Roma 2003.

${ }^{50}$ P. Gallarati, op. cit., pp. 180-183.

${ }^{51}$ F. Ceraolo, Addio del passato, in «Fata Morgana Web», 11 aprile 2021, $<$ https://www.fatamorganaweb.it/traviata-giuseppe-verdi-regia-martone-direzione-gatti/ (ultimo accesso 25/11/2021).

${ }^{52}$ Con tale espressione intendiamo la capacità del teatro di includere gli altri media in un ambiente fisico mantenendone la materialità. Su questo si veda C. Georgi, Liveness on Stage: Intermedial Challenges in Contemporary British Theatre and Performance, De Gruyter, Berlin-Boston 2014.
} 
nunciando al real time (e quindi al feedback $l o o p^{53}$ da esso procurato) gli spettacoli realizzati da Martone danno fondo compiutamene alle affordance tecnologiche del medium cinematografico, determinando una digital liveness mimetica e realistica, e dunque capace di un engagement ancora più robusto di quello «oscillante tra due principi [...] inconciliabili: mimesi e astrazione ${ }^{54}$ procurato dalla postura quasi “schizofrenica" della spettatorialità lirica.

La recente delocalizzazione delle messeinscena operistiche nello spazio narrativo e simbolico dei media elettronici ha assunto anche forme diverse, oltre alla rimediazione dei codici cinematografici o alla ricreazione di set filmici; in particolare, tre esperimenti di opera mediatizzata hanno imboccato la via drammaturgico-musicale del concerto di "pezzi" più o meno celebri, che, declinato con differenti modalità strutturali e fruitive ha attivato ulteriori gradienti di liveness nelle audience a distanza.

L'abbrivio di questo modello performativo nel frangente pandemico lo si deve al Teatro alla Scala che il 7 dicembre 2020, costretto a rinunciare al previsto allestimento dal vivo della Lucia di Lammermoor, ha scelto di affidarsi ancora una volta all'estro registico di Davide Livermore per confezionare uno show spiccatamente "multi-": testuale, mediale, fonico, narrativo. Preregistrato e poi trasmesso su Rai1 il pomeriggio dell'iconico appuntamento della Prima scaligera, lo spettacolo A riveder le stelle ha calamitato oltre 2 milioni di telespettatori, abbagliati da un rutilante programma ecosistemico che allineava 24 star della lirica nazionale e internazionale e si muoveva lungo una trentina di pezzi cantati (soprattutto arie, ma anche romanze e cavatine), cronologicamente e tematicamente eterogenei ma agganciati l'uno all'altro da una catena di "numeri" di danza e di brani in prosa e in versi - i primi eseguiti dal corpo di ballo del teatro, $\mathrm{i}$ secondi recitati da interpreti di vaglia della scena italiana.

Oggetto di pareri critici alquanto contrastanti ${ }^{55}$, l'iper-show scaligero ha avuto il merito di entrare in un territorio espressivo poco frequentato dalle produzioni mediatiz-

\footnotetext{
${ }^{53}$ Cfr. E. Fischer-Lichte, Estetica del performativo. Una teoria del teatro e dell'arte, Carocci, Roma 2014, p. 70.

${ }^{54}$ P. Gallarati, op. cit., p. 178.

${ }^{55}$ Si vedano ad esempio la netta stroncatura di Cesare Galla (cfr. C. Galla, op. cit.) e la review lusinghiera di Roberta Pedrotti: R. Pedrotti, Sant'Ambrogio e l'assenza, in "L'Ape musicale», 7 dicembre 2020, https://www.apemusicale.it/joomla/it/recensioni/56-opera/opera-2020/10808-milano-a-riveder-le-stelle-0712-2020 (ultimo accesso 25/11/2021).
} 
zate, ossia quello dell' «opera-mosaico, opera-catalogo, opera-atlante ${ }^{56}$, costruendo una ricca galleria di scene "aumentata" dalla tecnologia digitale e "modellata" dalla regia televisiva, che, a prescindere dalle valutazioni di gusto, risulta certamente portatrice di uno specifico gradiente di liveness. L'esperienza di fruizione mediale, in questo caso, si alimenta dello zapping percettivo-cognitivo tra differenti tipologie testuali, e si amplifica grazie alla convergenza tecnologica (sostenuta dalla "capienza semiotica" dell'opera lirica) dei numerosi linguaggi coinvolti: musica, canto, danza, prosa, video (le scenografie digitali realizzate da D-Wok), cinema (le tante e dirette -auto- citazioni filmiche, spesso riprese da pregresse regie di Livermore).

Un altro fattore determinante per l'applicabilità del concetto di liveness a questo spettacolo - in stretto rapporto con la sua fruizione televisiva - è dato dall'uso ampio e sistematico delle risorse espressive del découpage filmico.

Nonostante la diversità tra le tv-opere di Martone, che opacizzano la mediazione tecnologica per far risaltare il realismo scenico, e lo show ipermediale di Livermore, che «ribadisce il peso della tecnologia per la confezione dell'immaginario operistico ${ }^{57}$, in entrambi i casi, infatti, la strategia retorica dominante è la ritmizzazione del montaggio video in rapporto alla ritmizzazione testual-musicale. Sia Martone, che ha curato personalmente la regia televisiva de /l barbiere e de La traviata, sia l'équipe coordinata da Stefania Grimaldi, che si è occupata di quella di $A$ riveder le stelle, sfruttano a fondo i movimenti di macchina, i tagli delle inquadrature - con frequenti piani ravvicinati e reaction shot $-^{58}$, i punti di vista insoliti e creativi, sideralmente distanti da un'idea di ripresa neutra e soggettiva. La palpitante ritmizzazione di tali découpage televisivi (che naturalmente si aggiungono a quelli spettacolari) esalta la ritmizzazione propria dei testi operistici ${ }^{59}$, ma soprattutto coinvolge emotivamente chi guarda dal piccolo schermo, immergendolo in quella "corrente affettiva" di cui parla Auslander resa ancora più tra-

\footnotetext{
${ }^{56}$ G. Carluccio, S. Rimini, Ri-mappare l'Opera, in «Fata Morgana Web», 14 dicembre 2020, https://www.fatamorganaweb.it/a-riveder-le-stelle-livermore-chailly/ (ultimo accesso 25/11/2021).

57 Ibidem.

58 II cosidetto "piano di reazione" consiste in un rapido spostamento d'inquadratura sul viso di un personaggio per mostrare la sua reazione emotiva a ciò che si sente o che si vede.

${ }^{59}$ Con Lorenzo Bianconi chiamiamo testo operistico «il sistema composto da parola, musica e azione». Cfr. L. Bianconi, La regia d'opera: critica della critica, in «ll Saggiatore musicale», vol. XVII, n. 1, 2010, p. 105.
} 
scinante dalla "messa in ritmo" del montaggio, accuratamente studiata e realizzata prima della messa in onda ${ }^{60}$.

In particolare nella fruizione di spettacoli preregistrati, infatti, il dispositivo del découpage è cruciale: sia per modellare il senso drammaturgico - aggiungendo anche un sovrappiù di significato -, sia, come osservato da Michel Veilleux rispetto ai film d'opera televisivi girati da Pierre Jourdan negli anni Settanta, per «creare quel rapporto di prossimità e $d^{\prime}$ intimità con il personaggio che sulla scena è sovente assai difficile da instaurare ${ }^{61}$.

Una similare estetica del ritmo visivo, ma soprattutto una stessa «logica compositiva polifonica $»^{62}$ connota altre due sperimentazioni operistiche nate dalla crisi pandemica: lo spettacolo /l crepuscolo dei sogni del regista tedesco Johannes Erath, che il 25 gennaio 2021 è stato trasmesso in diretta streaming sulla web tv del Teatro Massimo di Palermo ottenendo più di 8.000 visualizzazioni, alle quali si sono aggiunte le oltre 22.000 registrate sulla pagina Facebook del teatro63; e l'opera "smart working" Alienati, ideata e prodotta dal Teatro Coccia di Novara e andata in onda il 2 giugno 2020 sulla piattaforma streaming OnTheatre con oltre 17.000 visualizzazioni ${ }^{64}$.

Ambedue i lavori si presentano come un fitto catalogo di brani lirici inanellati attorno a un plot originale: nel primo caso pezzi di repertorio distribuiti lungo un iter diegetico che, simbolicamente, procede "verso la luce" partendo da una situazione di crepuscolo; nel secondo una partitura di arie inedite e composte appositamente per il progetto, al quale tutti i partecipanti - compositori, librettista, regista, cantanti, tecnici del suono - hanno lavorato da remoto su piattaforme di web conferencing, appunto in smart working, facendo del paradigma produttivo dell'isolamento domestico l'elemento trainante della creazione artistica.

\footnotetext{
${ }^{60}$ A proposito dei video d'opera Emanuele Senici ha parlato di una " "messa in ritmo" per lo meno quadrupla: testo, musica, rappresentazione teatrale, video». Cfr. E. Senici, I/ video d'opera "dal vivo". Testualizzazione $e$ liveness nell'era digitale, in «ll Saggiatore musicale», vol. XVI, n. 2, 2009, p. 299.

${ }^{61}$ M. Veilleux, op. cit., p. 864.

62 Ibidem.

${ }^{63}$ Cfr. A. Foletto, Il crepuscolo dei sogni va forte sul web, "nessun lavoratore è stato escluso", in "la Repubblica», 29 gennaio 2021, https://www.repubblica.it/spettacoli/teatrodanza/2021/01/29/news/il_crepuscolo_dei_sogni_-284719591/ (ultimo accesso 25/11/2021).

${ }^{64}$ Cfr. Torna online "Alienati", l'opera in smart working del Coccia, in "NovaraToday», 11 luglio 2020, https://www.novaratoday.it/eventi/alienati-opera-smart-working-coccia.html (ultimo accesso 25/11/2021).
} 


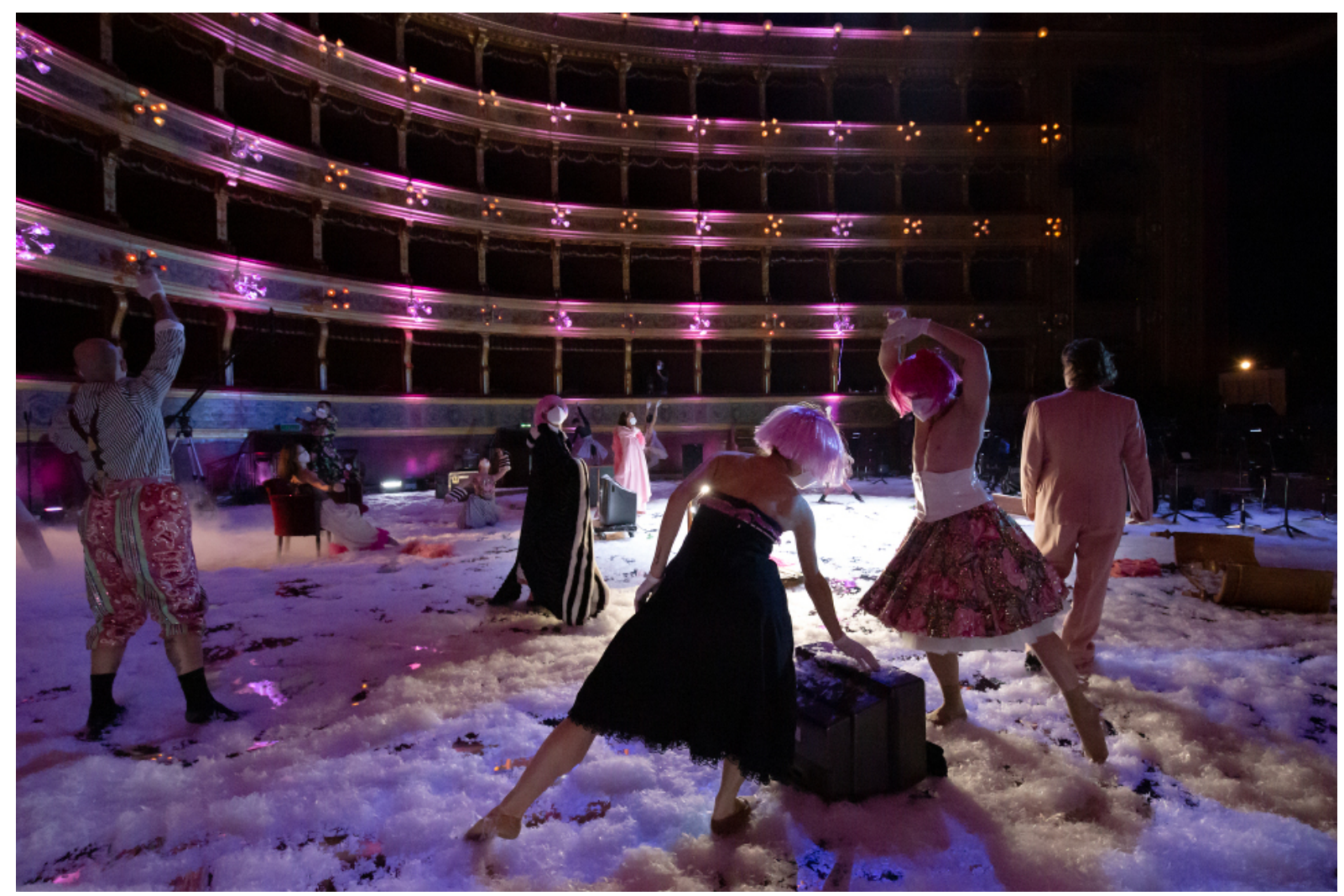

Fig. 03. Johannes Erath, Il crepuscolo dei sogni (2021).

Foto: Rossellina Garbo, per gentile concessione della Fondazione Teatro Massimo di Palermo.

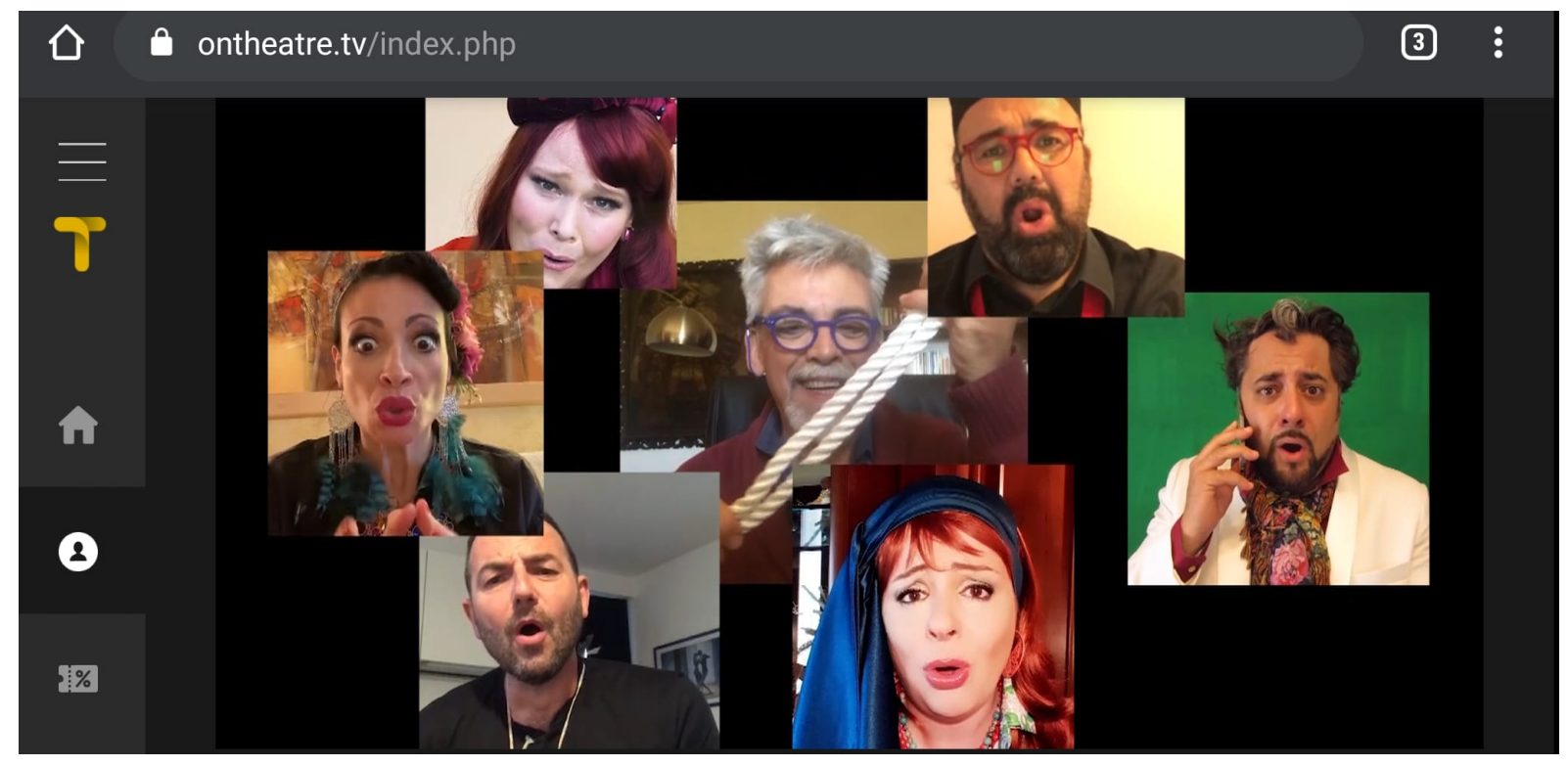

Fig. 04. Roberto Recchia, Alienati (2020).

Foto: immagine tratta dello streaming dello spettacolo, gentile concessione della Fondazione Teatro Coccia Onlus. 
La trama polifonica de I/ crepuscolo dei sogni, ovvero il format musicale "compilation di brani", diversamente da quella dello show scaligero preregistrato si snoda sulla temporalità "calda" e transeunte del live stream, conferendo alla multi-livellare mediatizzazione operistica un ulteriore e intenso grado di liveness. Esattamente, è la consapevolezza ${ }^{65}$ dell'azione in tempo reale dei cantanti, "ora ma non qui", che fa leva sul senso di liveness dell'audience; una consapevolezza che si incardina sulla «ridefinizione del concetto di presenza "qui e ora"», per cui l'apparizione live degli artisti agli occhi del pubblico connesso, benché "distanziata" dallo schermo, non nega affatto il concetto di presenza, ma lo rideclina come un «effetto di presenza di natura spiccatamente cognitiva e non fisica ${ }^{66}$.

Dal canto suo, invece, lo streaming di Alienati offre agli spettatori online una partitura composta da blocchi videomusicali assemblabili secondo opzioni definite da loro stessi, innescando uno stato di connessione con lo spettacolo davvero inedito, frutto della possibilità empirica di agire su di esso ${ }^{67}$, partecipando a un evento che può avvenire solo grazie alla loro (inter)attività.

In tal senso, la liveness del pubblico di Alienati scaturisce da una precisa «drammaturgia della partecipazione ${ }^{68}$ in cui la tecnologia digitale attiva un coinvolgimento secondo i modi del gaming, dell'esperienza del giocatore nel contesto dell'intrattenimento videoludico: lo spettatore online diventa un "player" che guida i cantanti, che costruisce insieme al resto dell'audience una trama narrativa partecipata, agendo all'interno di una drammaturgia procedurale subordinata alla sua creativa e concreta interazione. In questo modo, seguendo la prospettiva elaborata da Steve Dixon su quella che egli definisce «interactivity performance ${ }^{69}$, si vengono a creare effetti di «liveness aumentata», giacché l'uso delle webcam da parte degli artisti aumenta anziché diminuire le potenzialità

\footnotetext{
${ }^{65}$ Usiamo questo termine non casualmente ma in specifico riferimento a quanto affermato da Hans-Thies Lehmann a proposito della percezione dei corpi nelle performance digitali: "la presenza dal vivo non è in primis un fenomeno di realtà corporea, ma un fenomeno mentale, una questione di consapevolezza». Cfr. H.-T. Lehmann, La presenza a teatro, in E. Pitozzi (a cura di), op. cit., p. 17.

${ }^{66}$ A. Pizzo, op. cit., open edition: https://books.openedition.org/aaccademia/371\#bodyftn63 (ultimo accesso 25/11/2021).

${ }^{67}$ Sull'interattività dei pubblici connessi online si veda G. Boccia Artieri, Stati di connessione. Pubblici, cittadini e consumatori nella (Social) Network Society, FrancoAngeli, Milano 2012.

${ }^{68}$ Cfr. A. Lavender, Performance in the Twenty-first Century: Theatres of Engagement, Routledge, London \& New York 2016. Per un'attenta analisi di questo studio si rimanda a A. Pizzo, La drammaturgia della partecipazione nel mondo digitale, in «Mimesis Journal [Online]», vol. 6, n. 1, 2017, https://journals.openedition.org/mimesis/1200 (ultimo accesso 25/11/2021). ${ }^{69}$ S. Dixon, Digital performance, The MIT Press, Cambridge 2007, p. 4.
} 
della liveness, trasmettendo un' «idea di autenticità e di "realtà affidabile" »" su cui lo spettatore può agire personalmente.

Ecco allora che proprio da queste proposte di "streammare" l'opera lirica emerge un pieno dispiegamento delle potenzialità di liveness dell'online: espresse nell'uso delle piattaforme di streaming come «dispositivi che attivano una grammatica partecipativa forte ${ }^{71}$, nel tempo condiviso da una comunità di spettatori connessi simultaneamente (ora ma non qui), nell'interattività tecnologica ${ }^{72}$ che rimedia e potenzia l'interazione faccia a faccia, nella moltiplicazione digitale delle materie d'espressione che riattiva il senso di "dal vivo" dell'esperienza teatrale dell'opera nel segno di una fenomenologia più composita, e di un coinvolgimento più complesso.

Se il fulmineo sold out dell'imminente Prima scaligera dimostra la nostalgia per gli eventi in presenza, e il desiderio di tornare a godere di spettacoli tradizionali, conosciuti, tipici, altresì i numeri dei telespettatori e delle visualizzazioni online di questi esperimenti di opera in video indicano un posizionamento diverso dei pubblici della lirica, volto a forme spettacolari sperimentali, contemporanee, alternative, capaci di superare l'impasse dei teatri fisicamente inaccessibili e di produrre attraverso gli schermi nuovi e vividi gradienti di liveness.

\section{Riferimenti Bibliografici}

A. Amendola, V. Del Gaudio (a cura di), Teatro e immaginari digitali. Saggi di sociologia dello spettacolo multimediale, I gechi, Salerno 2017.

P. Auslander, Digital liveness: a historico-philosophical perspective, in «PAJ: A Journal of Performance and Art», vol. 34, n.3, 2012.

P. Auslander, Liveness: Performance in a Mediatized Culture [1999], Routledge, Oxon 2008.

C. Balme, Intermediality. Rethinking the relationship between theatre and media, in "The Wis. Zeitschrift der Gesellschaft für Theaterwissenschaft», n. 1, 2004, pp. 1-18.

C. Balme, The theatrical public sphere, Cambridge University Press, Cambridge 2014.

A. Balzola, A.M. Monteverdi, Le arti multimediali digitali, Garzanti, Milano 2004.

\footnotetext{
${ }^{70}$ S. Dixon, op. cit., p. 455.

${ }^{71} \mathrm{G}$. Boccia Artieri, L. Gemini, op. cit.

${ }^{72}$ Su questo concetto si veda L. Gemini, op. cit., 2003.
} 
M. Barker, Live at a cinema near you': How audiences respond to digital streaming of the arts, in J. Radbourne, H. Glow, K. Johanson (a cura di), The audience experience: A critical analysis of audiences in the performing arts, Intellect, Bristol 2013, pp. 15-34.

L. Bianconi, La regia d'opera: critica della critica, in «ll Saggiatore musicale», vol. XVII, n. 1, 2010, pp. 105- 118.

G. Boccia Artieri, L. Gemini, Per un live dei corpi a distanza, in «Doppiozero», $1^{\circ}$ maggio 2020, in https://www.doppiozero.com/materiali/un-live-dei-corpi-distanza (ultimo accesso 25/11/2021).

G. Boccia Artieri, Stati di connessione. Pubblici, cittadini e consumatori nella (Social) Network Society, FrancoAngeli, Milano 2012.

J.D. Bolter, R. Grusin, Remediation. Understanding New Media, The MIT Press, Cambridge 1999.

G. Carluccio, S. Rimini, Ri-mappare l'Opera, in "Fata Morgana Web», 14 dicembre 2020, in https://www.fatamorganaweb.it/a-riveder-le-stelle-livermore-chailly/ (ultimo accesso 25/11/2021).

F. Ceraolo, Addio del passato, in "Fata Morgana Web», 11 aprile 2021, in https://www.fatamorganaweb.it/traviata-giuseppe-verdi-regia-martone-direzionegatti/ (ultimo accesso 25/11/2021).

V. Del Gaudio, Modelli di serialità teatrale: Pratiche per un approccio mediologico al teatro a partire dai modelli seriali della Tragedia Endogonidia della Sociétas Raffaello Sanzio e Ada. Una cronaca familiare di Fanny \& Alexander, in «Mediascapes journal», n. 8, 2017, pp. 253-264.

M. De Marinis, In cerca dell'attore. Un bilancio del Novecento teatrale, Bulzoni, Roma 2000.

R. Debray, Vita e morte dell'immagine. Una storia dello sguardo in Occidente [1992], II Castoro, Milano 2010.

S. Dixon, Digital performance, The MIT Press, Cambridge 2007.

K. Edelman, B. Hurley, N. Gangopadhyay, Digital media trends survey, 14th edition, a cura di Deloitte's Technology, Media \& Telecommunications practice, in https://www2.deloitte.com/lu/en/pages/technology-mediaandtelecommunications/articles/digital-media-trends-survey.html (ultimo accesso 25/11/2021).

E. Fischer-Lichte, Estetica del performativo. Una teoria del teatro e dell'arte, Carocci, Roma 2014.

C. Galla, Opera lirica: fenomenologia dello streaming, in «Doppiozero», 12 dicembre 2020, in https://www.doppiozero.com/materiali/opera-lirica-fenomenologia-dello-streaming (ultimo accesso 25/11/2021).

P. Gallarati, Mimesi e astrazione nella regia del teatro musicale, in R. Alonge (a cura di), La regia teatrale: specchio delle brame della modernità, Edizioni di Pagina, Bari 2007, pp. 175-188. 
L. Gemini, S. Brilli, F. Giuliani, Il dispositivo teatrale alla prova del Covid-19. Mediatizzazione, liveness e pubblici, in «Mediascapes journal», n. 15, 2020, pp. 44-58.

L. Gemini, L'incertezza creativa. I percorsi sociali e comunicativi delle performance artistiche, FrancoAngeli, Milano 2003.

L. Gemini, Liveness: le logiche mediali nella comunicazione dal vivo, in "Sociologia della Comunicazione», n. 51, 2016, pp. 43-63.

C. Georgi, Liveness on Stage: Intermedial Challenges in Contemporary British Theatre and Performance, De Gruyter, Berlin-Boston 2014.

A. Gramsci, Letteratura e vita nazionale [1950], Editori Riuniti, Roma 1991.

W. Iser, L'atto della lettura. Una teoria della risposta estetica [1978], il Mulino, Bologna 1987.

J.-F. Jung, Le relâchement narratif dans le système de la captation, in Opera e televisione: un problema di linguaggi, Atti del Seminario internazionale (Roma, 27-30 ottobre 1993), I.R.Te.M., Roma 1997, pp. 39-55.

F. Krotz, The Meta-Process of 'Mediatisation' as a Conceptual Frame, in "Global media and communication», vol. 3, n. 3, 2007, pp. 256-260.

B. Large, voci Filming e Videotaping, in S. Sadie (a cura di), The New Grove Dictionary of Opera, Macmillan, London 1992.

A. Lavender, Performance in the Twenty-first Century: Theatres of Engagement, Routledge, London \& New York 2016.

H.-T. Lehmann, La presenza a teatro, in E. Pitozzi (a cura di), «Culture Teatrali. On Presence», n. 21, 2011, pp. 17-30.

L. Locatelli, Lo spettatore, in A. Cascetta, L. Peja (a cura di), Ingresso a teatro. Guida all'analisi della drammaturgia, Le Lettere, Firenze 2003, pp. 97-113.

L. Mango, La scrittura scenica. Un codice e le sue pratiche nel teatro del Novecento, Bulzoni, Roma 2003.

A.M. Monteverdi, Leggere uno spettacolo multimediale. La nuova scena tra video mapping, interaction design e Intelligenza Artificiale, Dino Audino, Roma 2020.

A.M. Monteverdi, Nuovi media, nuovo teatro. Teorie e pratiche tra teatro e digitalità, FrancoAngeli, Milano 2011.

S. O'Neil, J. Edelman, J. Sloboda, Opera Audiences and Cultural Value: A Study of Audience Experience, in «Creativeworks London Working Paper», n. 2, 2014.

A. Pizzo, La drammaturgia della partecipazione nel mondo digitale, in "Mimesis Journal [Online]», vol. 6, n. 1, 2017, in https://journals.openedition.org/mimesis/1200 (ultimo accesso 25/11/2021). 
A. Pizzo, Neodrammatico digitale: Scena multimediale e racconto interattivo, Accademia University Press, Torino 2013.

P.C. Rivoltella, La differenza comunicativa del teatro. Aspetti teorici e implicazioni educative, in «Comunicazioni Sociali», n. XVIII, 1996, pp. 5-29.

F. Rossi, L'opera italiana: lingua e linguaggio, Carocci, Roma 2018.

W. Schultz, Reconstructing Mediatisation as an Analytical Concept, in «European Journal of Communication», vol. 19, n. 1, 2004, pp. 87-101.

E. Senici, Il video d'opera "dal vivo". Testualizzazione e liveness nell'era digitale, in "ll Saggiatore musicale», XVI(2), 2009, pp. 273-312.

N. Till (a cura di), Opera Studies, Cambridge University Press, Cambridge 2012.

V. Valentini, Forme della presenza: performing arts e nuove tecnologie, in E. Pitozzi (a cura di), «Culture Teatrali. On Presence», n. 21, 2011, pp. 71-89.

G. Vattimo, La società trasparente, Garzanti, Milano 2000.

M. Veilleux, L'opera dal teatro allo schermo televisivo, in J.-J. Nattiez (a cura di), Storia della musica europea, Einaudi, Torino 2004, pp. 849-870.

M. Verdone, Teatro e cinema: interazioni, in C. Vicentini (a cura di), I/ teatro nella società dello spettacolo, il Mulino, Bologna 1983, pp. 51-62.

J. Whittaker, The Cyberspace Handbook, Routledge, London 2004.

\section{Sitografia}

https://www.artscouncil.org.uk/sites/default/files/download-

file/From_Live_to_Digital_OCT2016.pdf (ultimo accesso 25/11/2021).

https://www.teatromassimo.it/archivio/notizie/non-vi-lasciamo-senza-musica.html (ultimo accesso 25/11/2021).

https://www.youtube.com/user/operaroma (ultimo accesso 25/11/2021).

https://www.teatrosancarlo.it/it/pages/stage-at-home.html (ultimo accesso 25/11/2021).

https://www.teatroregio.torino.it/news/operaonthesofa (ultimo accesso 25/11/2021).

https://operastreaming.com/il-progetto/ (ultimo accesso 25/11/2021). 
Questo articolo nasce nell'ambito del progetto di ricerca del Dipartimento di Scienze Umanistiche dell'Università degli Studi di Catania ARIE - Audience, Remediation, Iconography, Environment in Contemporary Opera, di cui è responsabile scientifico la Prof.ssa Stefania Rimini (Università degli Studi di Catania).

\section{Biografia dell'autore/ Author's biography}

Laura Pernice (1988) è assegnista di ricerca presso il Dipartimento di Scienze Umanistiche dell'Università degli Studi di Catania nell'ambito del progetto ARIE - Audience, Remediation, Iconography, Environment in Contemporary Opera (diretto dalla Prof.ssa Stefania Rimini). Dottoressa di ricerca in Discipline dello Spettacolo all'Università di Catania, è autrice dei libri Motus. La vertigine multimediale (Villaggio Maori, 2016) e Giovanni Testori sulla scena contemporanea. Produzioni, regie, interviste (1993-2020) (Edizioni di Pagina, 2021). Dal 2015 è membro del comitato di redazione di «Arabeschi. Rivista internazionale di studi su letteratura e visualità.. Si occupa di teatro contemporaneo secondo prospettive di ricerca interdisciplinari, che coniugano i performing studies, la regia teatrale e la visual culture. Suoi articoli su registi e attori contemporanei (Giorgio Barberio Corsetti, Emma Dante, Fanny \& Alexander, Davide Livermore, Compagnia Lombardi-Tiezzi, Mario Martone, Motus, Teatro delle Albe, etc.) sono stati pubblicati in volumi collettanei e in riviste nazionali e internazionali.

Laura Pernice (1988) is Research Fellow at the University of Catania, Department of Humanities, within the project ARIE - Audience, Remediation, Iconography, Environment in Contemporary Opera (coordinated by Prof. Stefania Rimini). She holds a PhD in Disciplines of Performing Arts at the University of Catania, and she is author of the books Motus. La vertigine multimediale (Villaggio Maori, 2016) and Giovanni Testori sulla scena contemporanea. Produzioni, regie, interviste (1993-2020) (Edizioni di Pagina, 2021). From 2015 she is member of the editorial staff of «Arabeschi. Rivista internazionale di studi su letteratura e visualità». She studies contemporary theatre from an interdisciplinary research perspective, combining performing studies, theatre direction and visual culture. Her articles on contemporary directors and actors (Giorgio Barberio Corsetti, Emma Dante, Fanny \& Alexander, Davide Livermore, Compagnia Lombardi-Tiezzi, Mario Martone, Motus, Teatro delle Albe, etc.) have been published in collective volumes and national and international academic journals.

Articolo sottoposto a double-blind peer review 